\title{
A FOURIER INTEGRAL THEOREM FOR FUNCTIONS OF INTERVALS
}

\author{
W. F. NEWNS
}

1. Introduction. The persistence, under diverse hypotheses, of a reciprocal relationship between a point function and its Fourier transform suggests the possibility that something of the kind may also be true for functions of intervals. Perhaps the most symmetric form of the Fourier integral theorem is that due to Plancherel, as follows:

If $f \in L^{2}(0, \infty)$, then its Fourier cosine transform

$$
g(x)=\underset{a \rightarrow \infty}{\lim . \operatorname{m}}(2 / \pi)^{1 / 2} \int_{0}^{a} f(t) \cos x t d t
$$

exists as a limit in mean square and belongs to $L^{2}(0, \infty)$. Moreover,

$$
f(x)=\underset{a \rightarrow \infty}{\lim . \operatorname{los}}(2 / \pi)^{1 / 2} \int_{0}^{a} g(t) \cos x t d t,
$$

and

$$
\int_{0}^{\infty}\{f(x)\}^{2} d x=\int_{0}^{\infty}\{g(x)\}^{2} d x .
$$

If we wish to extend this theorem to functions of intervals, we need a suitable definition of Fourier cosine transform and a hypothesis analogous to $f \in L^{2}(0, \infty$.

Consider the special case $u(I)=\int_{I} f(t) d t$, where $f \in L^{2}$ and the integral is a Lebesgue integral. Then it is known $[2, \S 450$, p. 610] that

$$
\int_{a}^{b} \frac{\{u(I)\}^{2}}{|I|}=\lim _{\Delta S \rightarrow 0} \sum_{I \in S} \frac{\left\{\int_{I} f(t) d t\right\}^{2}}{|I|}=\int_{a}^{b}\{f(t)\}^{2} d t
$$

where $|I|$ denotes the length of the interval $I, \Delta S$ denotes the length of the longest interval $I$ in the subdivision $S$ of $(a, b)$ into nonoverlapping intervals, and the integral on the left is a Burkill integral [1].

Guided by this, we make the following definition:

Definition 1. A function $u$ defined on subintervals of $(0, \infty)$ is said to belong to the class $B^{2}$ if $u$ is integrable on every finite range and the

Received by the editors December 14, 1954. 
upper integral

$$
\int_{0}^{* \infty} \frac{\{u(I)\}^{2}}{|I|}=\lim _{\Delta \rightarrow \infty} \int_{0}^{* A} \frac{\{u(I)\}^{2}}{|I|}
$$

is finite.

Given a point function $f$ and a function $u$ of intervals, consider the sum $\sum_{k} f\left(t_{k}\right) u\left(I_{k}\right)$ taken over a subdivision $S$ of a finite interval $I_{0}$ into intervals $I_{k}$, and where $t_{k}$ is any point of $I_{k}$. If, as $\Delta S \rightarrow 0$, this sum tends to a limit, we may denote the limit by $\int_{I_{a}} f(t) u(I)$. Using this concept, we may now define Fourier transforms of functions of intervals.

Definition 2. The Fourier cosine transform of a function $u \in B^{2}$ is the function $g(x)=1.1 . \mathrm{m}_{\cdot a \rightarrow \infty}(2 / \pi)^{1 / 2} \int_{0}^{a} \cos x t u(I)$, where the limit is $a$ limit in mean square.

If we proceed heuristically, we could follow through an argument analogous to that given by Titchmarsh [3, pp. 436-438], and arrive at the following result:

TheOREM. If $u \in B^{2}$, then its Fourier cosine transform $g(x)$ exists and belongs to $L^{2}$ : in fact $\int_{0}^{\infty}\{g(x)\}^{2} d x \leqq \int_{0}^{* \infty}\{u(I)\}^{2} /|I|$. If $h$ is the (ordinary) Fourier cosine transform of $g$, then $\int_{0}^{x} u(I)=\int_{0}^{x} h(t) d t$ and $h(x)=u^{\prime}(x)$ p.p., where $u^{\prime}(x)$ is the derivative of $u(I)$.

Most of the foregoing is due to Dr. J. M. Whittaker, to whom I am indebted for suggesting this problem. The theorem stated is, in fact, true, but to justify the argument by which it was obtained would require the extension to Burkill integrals of a number of standard results on Lebesgue integrals. Because of the nonabsolute convergence of the Burkill integral and the lack of convenient criteria for the existence of integrals, these extensions have not been readily forthcoming, and we have preferred to proceed differently.

The inequality

$$
\int_{0}^{\infty}\{g(x)\}^{2} d x \leqq \int_{0}^{* \infty} \frac{\{u(I)\}^{2}}{|I|}
$$

can fail to be an equality. We shall see, however, that we always have

$$
\int_{0}^{\infty}\{g(x)\}^{2} d x=\int_{0}^{\infty} \frac{\{U(I)\}^{2}}{|I|},
$$

where $U\left(I_{0}\right)=\int_{I_{0}} u(I)$.

1 This departure from the usual notation for upper integrals is for typographical reasons. 


\section{Existence of the transform.}

2.1. Suppose that $f(t)$ is continuous, that $\int_{I_{0}}^{*}|u|<\infty$, and that $\sum_{k} f\left(t_{k}\right) u\left(I_{k}\right)$ converges, as $\Delta S \rightarrow 0$, for some definite choice of $t_{k}$ for each $I_{k}$. Then it converges for every choice of $t_{k}$, i.e. the transform $\int_{I_{a}} f(t) u(I)$ exists.

Here, and throughout this section, $I_{0}$ denotes a finite subinterval of $(0, \infty)$ and $S$ a subdivision of $I_{0}$ in to intervals $I_{k}$, the length of the longest of which is $\Delta S$.

Let $l$ be the limit of $\sum_{k} f\left(t_{k}\right) u\left(I_{k}\right)$. Then given $\epsilon>0$, we can find $\delta>0$ such that for any subdivision $S$ for which $\Delta S \leqq \delta$,

$$
\begin{gathered}
\left|\sum_{k} f\left(t_{k}\right) u\left(I_{k}\right)-l\right| \leqq \epsilon / 2, \\
\sum_{k}\left|u\left(I_{k}\right)\right| \leqq 1+\int_{I_{0}}^{*}|u|, \\
\left|f(t)-f\left(t^{\prime}\right)\right| \leqq \epsilon /\left(2+2 \int_{I_{0}}^{*}|u|\right) \quad \text { for }\left|t-t^{\prime}\right| \leqq \delta .
\end{gathered}
$$

Hence if $t_{k}^{\prime}$ is any point of $I_{k}$,

$$
\left|\sum_{k} f\left(t_{k}^{\prime}\right) u\left(I_{k}\right)-\sum_{k} f\left(t_{k}\right) u\left(I_{k}\right)\right| \leqq \epsilon / 2,
$$

and hence $\left|\sum_{k} f\left(t_{k}^{\prime}\right) u\left(I_{k}\right)-l\right| \leqq \epsilon$, which proves our assertion.

2.2. If $f(I) \rightarrow f(t)$ as $I \rightarrow t, f(t)$ is continuous and $\int_{I_{0}^{*}}^{*}|u|<\infty$, then if $\int_{I_{0}} f(I) u(I)$ exists so does $\int_{I_{0}} f(t) u(I)$, and the two are equal.

By $f(I) \rightarrow f(t)$ as $I \rightarrow t$, we mean that for each $t$, given $\epsilon>0$, we can find $\delta>0$ such that $|f(I)-f(t)| \leqq \epsilon$ whenever $t \in I$ and $|I| \leqq \delta$. The function $f(I)-f(t)$, where $t$ is the mid-point of $I$, is therefore a continuous function of intervals. By the uniform continuity theorem, given $\epsilon>0$, we can find $\delta>0$ such that $|f(I)-f(t)| \leqq \epsilon$ provided $|I| \leqq \delta$. Hence, for small $\Delta S$,

$$
\begin{aligned}
\left|\sum_{k} f\left(I_{k}\right) u\left(I_{k}\right)-\sum_{k} f\left(t_{k}\right) u\left(I_{k}\right)\right| & \leqq \epsilon \sum_{k}\left|u\left(I_{k}\right)\right| \\
& \leqq \epsilon\left(1+\int_{I_{0}}^{*}|u|\right),
\end{aligned}
$$

and the rest follows from 2.1 .

2.3. Suppose that $u$ is absolutely continuous and integrable, and that $f(t)$ is continuous. Then $\int_{I_{0}} f(t) u(I)$ exists: in fact

$$
\int_{I_{0}} f(t) u(I)=\int_{I_{0}} f(I) u(I)=\int_{I_{0}} f(t) u^{\prime}(t) d t,
$$

where $f(I) \cdot|I|=\int_{I} f(t) d t$. 
For since $f(I)$ is bounded, $f(I) u(I)$ is absolutely continuous [1, p. 287]. Moreover, since $f(I) \rightarrow f(t)$ as $I \rightarrow t$ and $u$ is differentiable p.p. [1, Theorem 7.2], $f(I) u(I)$ is differentiable p.p., and its derivative is $f(t) u^{\prime}(t)$. Hence $f(I) u(I)$ is in tegrable $\left[1\right.$, Theorem 7.6] and $\int_{I_{a}} f(I) u(I)$ $=\int_{I_{a}} f(t) u^{\prime}(t) d t$. Finally, $\int_{I_{0}^{*}}^{*}|u|<\infty$ since $u$ is absolutely continuous $[1$, Theorem 3.6], so that the rest follows from 2.2 .

\section{Functions of class $B^{2}$.}

3.1. If $u \in B^{2}$, then $u$ is absolutely continuous on any finite interval. For, using Schwarz's inequality, if $\sum_{k}\left|I_{k}\right|$ is sufficiently small,

$$
\begin{aligned}
\sum_{k}\left|u\left(I_{k}\right)\right| & \leqq\left(\sum_{k} \frac{\left\{u\left(I_{k}\right)\right\}^{2}}{\left|I_{k}\right|} \cdot \sum_{k}\left|I_{k}\right|\right)^{1 / 2} \\
& \leqq\left(1+\int_{I_{0}}^{*} \frac{\{u(I)\}^{2}}{|I|}\right)^{1 / 2} \cdot\left(\sum_{k}\left|I_{k}\right|\right)^{1 / 2} .
\end{aligned}
$$

3.2. If $u \in B^{2}$ and $U\left(I_{0}\right)=\int_{I_{0}} u(I)$, then $U \in B^{2}$. Moreover, $u^{\prime}(x) \in L^{2}$ and

$$
\int_{0}^{\infty}\left\{u^{\prime}(x)\right\}^{2} d x=\int_{0}^{\infty} \frac{\{U(I)\}^{2}}{|I|} \leqq \int_{0}^{* \infty} \frac{\{u(I)\}^{2}}{|I|} .
$$

Since $U$ is absolutely continuous and additive, we have $[1$, Theorems 2.1 and 7.6, Corollary] $U\left(I_{k}\right)=\int_{I_{k}} U^{\prime}(x) d x$ for any $I_{k}$. Hence, for any subdivision $S$ of $I_{0}$,

$$
\sum_{k} \frac{\left\{U\left(I_{k}\right)\right\}^{2}}{\left|I_{k}\right|}=\sum_{k} \frac{\left\{\int_{I_{k}} U^{\prime}(x) d x\right\}^{2}}{\left|I_{k}\right|} .
$$

But, by Schwarz's inequality [1, p. 282],

$$
\frac{\left\{U\left(I_{k}\right)\right\}^{2}}{\left|I_{k}\right|}=\frac{\left\{\int_{I_{k}} u(I)\right\}^{2}}{\left|I_{k}\right|} \leqq \int_{I_{k}}^{*} \frac{\{u(I)\}^{2}}{|I|} \cdot \frac{\int_{I_{k}}|I|}{\left|I_{k}\right|}=\int_{I_{k}}^{*} \frac{\{u(I)\}^{2}}{|I|}
$$

and hence the sum on the left of (2) does not exceed

$$
\int_{I_{0}}^{*} \frac{\{u(I)\}^{2}}{|I|}
$$

since upper integrals decrease on subdivision. It follows [2, \$450] that as $\Delta S \rightarrow 0$ the sums in (2) must converge to $\int_{I_{0}}\left\{U^{\prime}(x)\right\}^{2} d x$ and that this integral does not exceed the upper integral (3). Thus 


$$
\int_{I_{0}} \frac{\{U(I)\}^{2}}{|I|}
$$

exists and is bounded for $I_{0} \subset(0, \infty)$, so that $U \in B^{2}$. Since, moreover, $u^{\prime}(x)=U^{\prime}(x)$ p.p., we have $u^{\prime}(x) \in L^{2}$ and

$$
\int_{0}^{\infty}\left\{u^{\prime}(x)\right\}^{2} d x=\int_{0}^{\infty} \frac{\{U(I)\}^{2}}{|I|} \leqq \int_{0}^{\infty} \int_{I}^{*} \frac{\{u(I)\}^{2}}{|I|} \leqq \int_{0}^{* \infty} \frac{\{u(I)\}^{2}}{|I|}
$$

4. Proof of the theorem. By 3.1 and 2.3, the integral $\int_{0}^{a} \cos x t u(I)$ exists and is equal to $\int_{0}^{a} \cos x t u^{\prime}(t) d t$. Hence the Fourier cosine transform $g(x)$ of the function $u(I)$ is the same as the transform of $u^{\prime}(x)$, which exists, by 3.2 and Plancherel's theorem. Thus $g \in L^{2}, h \in L^{2}$ and $h(x)=u^{\prime}(x)$ p.p. Also, using 3.2,

$$
\int_{0}^{\infty}\{g(x)\}^{2} d x=\int_{0}^{\infty}\left\{u^{\prime}(x)\right\}^{2} d x \leqq \int_{0}^{* \infty} \frac{\{u(I)\}^{2}}{|I|}
$$

and, since $u$ is absolutely continuous,

$$
\int_{0}^{x} u(I)=\int_{0}^{x} u^{\prime}(t) d t=\int_{0}^{x} h(t) d t
$$

5. Example. Define a point function $f(x)$ as follows: if $0 \leqq x \leqq 1$, then $f$ coincides with the continuous, monotone increasing but nonabsolutely continuous function defined by Titchmarsh $[3,11.72$, p. 366]; and if $x>1$, we define $f(x)=1$. Then $f(0)=0, f(x)=1$ for $x \geqq 1$ and $f^{\prime}(x)=0$ p.p. For $I=(a, b)$, define

$$
u(I)=\{(f(b)-f(a))|I|\}^{1 / 2} .
$$

Then $u$ is absolutely continuous; for, by Schwarz's inequality,

$$
\sum_{k}\left|u\left(I_{k}\right)\right| \leqq\left\{\sum_{k}\left(f\left(b_{k}\right)-f\left(a_{k}\right)\right) \sum_{k}\left|I_{k}\right|\right\}^{1 / 2} \leqq\left\{\sum_{k}\left|I_{k}\right|\right\}^{1 / 2} \text {. }
$$

Moreover, $u$ is differentiable p.p. and its derivative is

$$
\lim _{I \rightarrow x}\left\{\frac{f(b)-f(a)}{|I|}\right\}^{1 / 2}=\left\{f^{\prime}(x)\right\}^{1 / 2}=0
$$

Hence $u$ is integrable, and since $\{u(I)\}^{2} /|I|=f(b)-f(a)$ is additive

$$
\int_{0}^{X} \frac{\{u(I)\}^{2}}{|I|}=f(X)-f(0)=1
$$

for $X \geqq 1$, and 


$$
\int_{0}^{\infty} \frac{\{u(I)\}^{2}}{|I|}=1 .
$$

Hence $u \in B^{2}$. However

$$
\begin{aligned}
U(I) & =\int_{I} u(I)=\int_{a}^{b} u^{\prime}(x) d x=0, \\
\frac{\{U(I)\}^{2}}{|I|} & =0, \text { and } \int_{0}^{\infty} \frac{\{U(I)\}^{2}}{|I|}=0<1=\int_{0}^{\infty} \frac{\{u(I)\}^{2}}{|I|} .
\end{aligned}
$$

6. Special cases. If we take $u(I)=\int_{I} f(t) d t$, where $f \in L^{2}$, then the theorem reduces to Plancherel's theorem, equality holding in (1) since $u$ is additive and hence $U(I)=u(I)$. Similarly, a suitable choice of $u$ $[1$, p. 281 , no. 4$]$ would give a version involving Riemann-Stieltjes instead of Lebesgue integrals.

A rather unexpected result emerges, however, if we make the following choice for $u\left[1\right.$, p. 280 , no. 2]: for any interval $I=\left(y, y^{\prime}\right)$, we define $u(I)=y m E$, where $m E$ is the measure of the set $E=\{x: y$ $\left.\leqq f(x)<y^{\prime}\right\}, f$ being a function defined and integrable on some interval $(a, b)$. We have

$$
\int_{-\infty}^{\infty} u(I)=\int_{a}^{b} f(x) d x, \quad \int_{0}^{\infty} u(I)=\int_{a}^{b} f^{+}(x) d x,
$$

where $f^{+}(x)$ is $f(x)$ if $f(x)>0$ and zero otherwise; and

$$
\int_{0}^{X} u(I)=\int_{a}^{b}\left[f^{+}(X)\right]_{x} d x
$$

where $[y]_{x}$ is $y$ if $y<X$ and zero otherwise.

In general, we shall not have $u \in B^{2}$ : for example, if $f(x)=1$ for all $x, u(I)=0$ if $1 \notin I$ and $u(I)=y(b-a)$ if $1 \in I=\left(y, y^{\prime}\right)$, so that $u$ is not continuous. We see from this that $u \in B^{2}$ implies that $f$ is constant on no set of positive measure. Let us suppose for simplicity that $f$ is strictly monotone. Then $E$ is an interval $\left(x, x^{\prime}\right)$, and

$$
\begin{aligned}
\int_{0}^{x} \cos \xi t u(I) & =\lim _{\Delta S \rightarrow 0} \sum_{k} \cos \xi y_{k} \cdot y_{k}\left(x_{k+1}-x_{k}\right) \\
& =\int_{a}^{b}\left[f^{+}(x)\right]_{x} \cos \xi f(x) d x
\end{aligned}
$$

the latter being a Riemann integral. Thus the Fourier cosine transform of $u$ is here 


$$
g(x)=(2 / \pi)^{1 / 2} \int_{a}^{b} f^{+}(t) \cos x f(t) d t .
$$

If $h$ is the ordinary transform of $g$, we have

$$
\begin{aligned}
\int_{0}^{x} h(t) d t & =(2 / \pi)^{1 / 2} \int_{0}^{\infty} x^{-1} \sin x X \cdot g(x) d x=\int_{0}^{x} u(I) \\
& =\int_{a}^{b}\left[f^{+}(t)\right]_{X} d t,
\end{aligned}
$$

a formula which may also be verified directly.

\section{REFERENCES}

1. J. C. Burkill, Functions of intervals, Proc. London Math. Soc. (2) vol. 22 (1924) pp. 275-310.

2. E. W. Hobson, Theory of functions of a real variable, vol. 1, 2d ed., Cambridge University Press 1921.

3. E. C. Titchmarsh, The theory of functions, 2d ed., Oxford University Press, 1939.

UNIVERSITY OF LIVERPOOL 